\title{
CONTINUED FRACTIONS OF ARITHMETIC SEQUENCES OF QUADRATICS
}

\author{
MENNY AKA
}

\begin{abstract}
Let $x$ be a quadratic irrational and let $\mathbb{P}$ be the set of prime numbers. We show the existence of an infinite set $S \subset \mathbb{P}$ such that the statistics of the period of the continued fraction expansions along the sequence $\{p x: p \in S\}$ approach the 'normal' statistics given by the Gauss-Kuzmin measure. Under the generalized Riemann hypothesis, we prove that there exist full density subsets $S \subset \mathbb{P}$ and $T \subset \mathbb{N}$ satisfying the same assertion. We give a rate of convergence in all cases.
\end{abstract}

\section{INTRODUCTION}

In this paper we address some natural questions that were raised in [2] on the study of the evolution of the continued fraction expansion within a fixed real quadratic field. The reader is referred there for more details and motivation. We review here the necessary definitions for the presentation of our results.

For a real number $x \in[0,1] \backslash \mathbb{Q}$ let $x=\left[a_{1}(x), a_{2}(x), \ldots\right]$ be its uniquely defined continued fraction expansion. For a finite sequence of natural numbers $w=\left(w_{1}, \ldots, w_{k}\right)$ we define the frequency of appearance of the pattern $w$ in the continued fraction expansion of $x$, by

$$
D(x, w)=\lim _{N} \frac{1}{N} \#\left\{1 \leq n \leq N: w=\left(a_{n+1}(x), \ldots, a_{n+k}(x)\right)\right\} .
$$

We make the convention that for $x \in \mathbb{R} \backslash \mathbb{Q}, D(x, w):=D(\bar{x}, w)$ where $\bar{x} \in[0,1]$ and $\bar{x}=x \bmod 1$. As we explain in the introduction of [2], for Lebesgue almost any $x, D(x, w)$ exists and is equal to some explicit integral which depends only on $w$. We denote this almost sure value as $c_{w}$.

In 2] we fix a quadratic irrational $x$ and study the behavior of $D\left(p^{n} x, w\right)$ as $n \rightarrow \infty$ where $p$ is a fixed natural number, or more generally, the behavior of $D\left(p_{n} x, w\right)$ when $p_{n} \rightarrow \infty$ are supported on a fixed finite set of primes $S$ (by supported we mean that if a prime $p$ divides $p_{n}$ for some $n$ then $p \in S$ ). The methods of [2] fail when the numbers $p_{n}$ are not supported of finite set of primes. The aim of this note is merely to rephrase known results in analytic number theory using a refinement of Duke's Theorem (See section 2.1) in order to answer question (4) of [2, §2.7] and some related results. Using the results of [4, 16] we have the following unconditional result:

Theorem 1. There exist at most two real quadratic fields such that if a quadratic irrational $x$ does not belong to them, there exist an infinite subset 
$S \subset \mathbb{P}$ and $\delta>0$ such that for any pattern $w$ there exists a constant $C=$ $C(w, x)$ with

$$
p \in S \Longrightarrow\left|D(p x, w)-c_{w}\right|<C p^{-\delta} .
$$

Using [14] with with [11, 17], we have the following two conditional results:

Theorem 2. Let $x$ be a quadratic irrational. Then, assuming that the Generalized Riemann Hypothesis (GRH) holds, there exist a density one subset $S \subset \mathbb{N}$ and $\delta>0$ such that for any pattern $w$ there exists a constant $C=C(w, x)$ with

$$
m \in S \Longrightarrow\left|D(m x, w)-c_{w}\right|<C m^{-\delta} .
$$

Similarly, let $P$ denote the set of primes. Assuming that the GRH holds, there exist a density one sequence $S \subset P$ and $\delta>0$ such that for any pattern $w$ there exists a constant $C=C(w, x)$ with

$$
p \in S \Longrightarrow\left|D(p x, w)-c_{w}\right|<C p^{-\delta} .
$$

Remark 3. Using the same methods, Theorems 1 and 2 can be slightly generalized. For example, these theorems remain valid if we can consider $D\left(w, \frac{x}{m}\right)$ instead of $D(w, m x)$ for $m$ coprime with $\operatorname{disc}(\mathbb{Q}(\mathrm{x}))$ (as the associated orders are the same, i.e. $\mathcal{O}_{m x}=\mathcal{O}_{\frac{x}{m}}$, see $\$ 2.1$ ). Theorems 1 and 2 should be viewed as what we consider to be representative results that can be achieved via these techniques.

1.1. Organization of this note. As mentioned above the following note is merely a reformulation of known results in analytic number theory. As in 2] the above results follow from equidistribution of geodesic loops on the modular surface. In this context, there is an arithmetically defined sequence of collections $\mathcal{G}_{d}$ of closed geodesics that equidistribute as $d \rightarrow \infty$; this statement is now classically known as "Duke's Theorem". When one considers the statistics of continued fraction sequence along some arithmetic sequences, one is led to consider subcollections (in fact we will consider one "long" geodesic) of $\mathcal{G}_{d}$. When these subcollections occupy a substantial part of $\mathcal{G}_{d}$, one can hope that they will equidistribute too (c.f. the far-reaching conjecture [6. Conjecture 1.11]). Section 2 is devoted to describe these notions and state a variant of Duke's Theorem for subcollections. This establishes the bridge between statistics of continued fractions expansion and closed geodesics. Section 3 starts with the simple observations connecting the later to orders of certain matrices. Then we state several results from analytic number theory about these orders (which can be viewed as analogues of Artin's conjecture on primitive roots) and deduce our results.

Acknowledgments. This work emerged from a joint work with Uri Shapira and I wish to thank him for many discussions on this topic and his interests in this project. We would like to thank Lior Rosenzweig to referring him to Par Kurlberg's work and to Paul Nelson and Philippe Michel for fruitful discussions. Many thanks are due to Andreas Wieser for a careful reading 
of this note, and to Alain Valette for encouraging the publication of this manuscript. Thanks are due to the anonymous referee for improving the exposition. We acknowledge the support of Advanced Research Grant 228304 from the European Research Council.

\section{Preliminaries}

We freely use notions from algebraic number theory in the context of quadratic real extensions, as orders, discriminant, regulators, etc. The reader is referred to [5, Page 133] or any algebra number theory book for background on these notions.

As in [2, the above theorem follows from equidistribution theorems of certain geodesic loops on the modular surface so we briefly record the relevant settings. For the geodesic flow on the modular surface and also for its relation to continued fractions, we refer the reader to [8, Section 9.6]. Let $X_{2}=$ $\mathrm{SL}_{2}(\mathbb{Z}) \backslash \mathrm{SL}_{2}(\mathbb{R})=: \Gamma \backslash G$ be the unit tangent bundle of the modular surface. The geodesic flow on $X_{2}$ is given by the action of the diagonal group

$$
A:=\left\{a_{t}:=\left(\begin{array}{cc}
e^{\frac{-t}{2}} & 0 \\
0 & e^{\frac{t}{2}}
\end{array}\right)\right\}_{t \in \mathbb{R}}<G
$$

sending $\Gamma g$ to $\Gamma g a_{t}^{-1}$. An element $x \in \mathbb{R}$ is called quadratic irrational if $\mathbb{Q}(x)$ is a real quadratic field. For a quadratic irrational $x$, we let

$$
g_{x}= \begin{cases}\frac{1}{\sqrt{x-\bar{x}}}\left(\begin{array}{cc}
x & \bar{x} \\
1 & 1
\end{array}\right) & \text { if } x-\bar{x}>0 \\
\frac{1}{\sqrt{\bar{x}-x}}\left(\begin{array}{cc}
\bar{x} & x \\
1 & 1
\end{array}\right) & \text { if } x-\bar{x}<0\end{cases}
$$

and set $L_{x}=\Gamma g_{x} A$. It is known that it is a closed geodesic in $X_{2}$ (see [2, Lemma 8.2] ).

2.1. Duke's Theorem for subcollections. Proofs of all the statement of this subsection can be found in [7, §2] and the references within). For any closed geodesic $L$ one can choose a quadratic irrational $x$ such that $L=L_{x}$. We can associate with $L_{x}$ an order in $\mathbb{Q}(x)$ by $\mathcal{O}_{x}:=\left\{a: a \Lambda_{x} \subseteq \Lambda_{x}\right\}$ where $\Lambda_{x}:=\mathbb{Z} \cdot 1 \oplus \mathbb{Z} \cdot x$. This order does not depend on the choice of $x$ and therefore for any geodesic loop $L=L_{x}$ we can associate a discriminant by $\operatorname{Disc}(L)=\operatorname{Disc}\left(\mathcal{O}_{x}\right)$. Let $\mathcal{G}_{D}$ be the set of geodesic loops which have discriminant $D$.

Theorem 4. Let $D$ be positive non-square discriminant and $\mathcal{O}_{D}:=\mathbb{Z}\left[\frac{D+\sqrt{D}}{2}\right]$ be the order of discriminant $D$. We have:

(1) $\left|\mathcal{G}_{D}\right|=\left|\operatorname{Pic}\left(\mathcal{O}_{D}\right)\right|$ where $\operatorname{Pic}\left(\mathcal{O}_{D}\right)$ is the ideal class group of $\mathcal{O}_{D}$.

(2) The length of any $L$ in $\mathcal{G}_{D}$ is equal to $\operatorname{Reg}\left(\mathcal{O}_{D}\right)$, the regulator of $\mathcal{O}_{D}$.

(3) The total length of the collection $\mathcal{G}_{D}$ is $\operatorname{Reg}\left(\mathcal{O}_{D}\right)\left|\mathcal{G}_{D}\right|=D^{\frac{1}{2}+o(1)}$. 
Proof. See [7, §2].

For any $L \in \mathcal{G}_{D}$ we let $\mu_{L}$ be the normalized length measure supported on $L$ and we let $l(L)$ denote the length of $L$. By Theorem 4 , we have for $L \in \mathcal{G}_{D}$ that $l(L)=\operatorname{Reg}\left(\mathcal{O}_{D}\right)$.

Theorem 5. There exist $a \delta>0$ with the following property: Let $\left\{L_{i}\right\}$ be a sequence of geodesics with $l\left(L_{i}\right)=\operatorname{Reg}\left(L_{i}\right)>\operatorname{Disc}\left(L_{i}\right)^{\frac{1}{2}-a}$ for some $0 \leq a<\delta$. Then for any $f \in C_{c}^{\infty}(X)$ we have

$$
\left|\mu_{L_{i}}(f)-\mu_{X_{2}}(f)\right| \ll S(f) \operatorname{Disc}\left(L_{i}\right)^{a-\delta}
$$

where $S(f)$ is a Sobolev norm.

Proof. When $\left\{\operatorname{Disc}\left(L_{i}\right)\right\}_{i=1}^{\infty}$ are fundamental discriminants (i.e. square-free) this theorem follows from [17, Theorem 6.5.1] or [11, Theorem 6]. In [10, Corollary 1.4] one finds an explicit exponent $\delta=\frac{1}{2827}$. For the results of this paper, we are mainly in non-fundamental discriminants. Unfortunately, such a result does not exist in print. Nevertheless it is knows to experts (see e.g. [15]) and it will appear in an upcoming monograph by Philippe Michel. The reader is also referred to [1] for a related result and general discussion of such results.

\section{PROOFS}

Let $D>2$ be a fundamental discriminant, $\mathcal{O}_{D}$ the unique order of discriminant $D$ and $K=\operatorname{Frac}\left(\mathcal{O}_{D}\right)$. Hereafter, we make the explicit choice of $x_{D}=\sqrt{D}$ when $D \neq 1(\bmod 4)$ and $x_{D}=\frac{1+\sqrt{D}}{2}$ when $D=1(\bmod 4)$ so $\mathbb{Z}\left[x_{D}\right]=\mathcal{O}_{D}$.

Lemma 6. For any integer $N \in \mathbb{N}$, the discriminant of the order $\mathbb{Z}\left[N x_{D}\right]$ is $N^{2} D$.

Proof. It is well known that for any order $\mathcal{O} \subset \mathcal{O}_{K}$,

$$
\operatorname{Disc}(\mathcal{O})=\operatorname{Disc}\left(\mathcal{O}_{K}\right)\left(\mathcal{O}_{K}: \mathcal{O}\right)^{2} \text {. }
$$

As $\left[\mathbb{Z}\left[x_{D}\right]: \mathbb{Z}\left[N x_{D}\right]\right]=N$ the lemma follows.

As there is a unique order of a given (not necessarily fundamental) discriminant, we conveniently denote $\mathcal{O}_{N^{2} D}:=\mathbb{Z}\left[N x_{D}\right]$ the order of discriminant $N^{2} D$.

3.1. Identifying $\mathcal{O}_{D}$ with $\mathbb{Z}^{2}$. Using the basis $B=\left\{1, x_{D}\right\}$ of $K$ we identify $K$ with $\mathbb{Q}^{2}$ and $\mathcal{O}_{D}$ with $\mathbb{Z}^{2}$. For $\alpha \in K$ we write $m_{\alpha}$ as the multiplication map by $\alpha$ on $K$. Writing $m_{\alpha}$ in the basis $B$ (using rows), we get an embed$\operatorname{ding} \varphi: K \rightarrow M_{2}(\mathbb{Q})$. The reader can readily verify that $\varphi^{-1}\left(M_{2}(\mathbb{Z})\right)=\mathcal{O}_{D}$, and that for all $\alpha \in K$, we have $\operatorname{Trace}_{\mathrm{K} / \mathbb{Q}}(\alpha)=\operatorname{Trace}(\varphi(\alpha)), \operatorname{Norm}_{\mathrm{K} / \mathbb{Q}}(\alpha)=$ $\operatorname{Det}(\varphi(\alpha))$.

Lemma 7. For any $\alpha \in \mathcal{O}_{D}$ we have $\alpha \in \mathcal{O}_{N^{2} D}$ if and only if $\varphi(\alpha)$ is a scalar matrix modulo $N$. 
Proof. By definition $\varphi(\alpha)=\left(\begin{array}{ll}P & Q \\ R & S\end{array}\right)$ where $\alpha=P+Q x_{D}, \alpha x_{D}=R+S x_{D}$. Thus if $\varphi(\alpha)$ is a scalar matrix modulo $N$, in particular $Q=0(\bmod N)$, so $\alpha \in \mathcal{O}_{N^{2} D}$.

Now, let $\alpha \in \mathcal{O}_{N^{2} D}=\mathbb{Z}\left[N x_{D}\right]$, and write $\alpha=a+b N x_{D}$ for some $a, b \in \mathbb{Z}$. As $\varphi$ is a ring-homomorphism, we have

$$
\varphi(\alpha)=a I_{2}+b N \varphi\left(x_{D}\right)
$$

where $I_{2}$ is the identity matrix. As $x_{D}$ is integral, $\varphi\left(x_{D}\right) \in M_{2}(\mathbb{Z})$ and therefore $\varphi(\alpha)=a I_{2}$ modulo $N$.

Corollary 8. Let $\epsilon=\epsilon_{D}$ be a fundamental unit of $\mathcal{O}_{D}$ and for any integer $N \in \mathbb{N}$, let $R(N)=\min \left\{k: \varphi\left(\epsilon_{D}\right)^{k}= \pm I\right.$ modulo $\left.N\right\}$. We have

$$
\operatorname{Reg}\left(\mathcal{O}_{N^{2} D}\right)=\operatorname{Reg}\left(\mathcal{O}_{D}\right) R(N) \text {. }
$$

It follows that $\operatorname{Reg}\left(\mathcal{O}_{N^{2} D}\right) \gg \operatorname{ord}_{\mathrm{N}}(\varphi(\epsilon))$ where $\operatorname{ord}_{\mathrm{N}}(\varphi(\epsilon))$ is the order of the reduction $\bmod N$ of $\varphi(\epsilon)$ in $\mathrm{SL}_{2}(\mathbb{Z} / N \mathbb{Z})$.

Proof. By definition of the regulator, we have that

$$
\operatorname{Reg}\left(\mathcal{O}_{N^{2} D}\right)=\operatorname{Reg}\left(\mathcal{O}_{D}\right) \cdot\left(\mathcal{O}_{D}^{\times}: \mathcal{O}_{N^{2} D}^{\times}\right),
$$

so we need to show that

$$
\left(\mathcal{O}_{D}^{\times}: \mathcal{O}_{N^{2} D}^{\times}\right)=R(N) .
$$

Using Lemma 7 we have that $\epsilon^{k} \in \mathcal{O}_{N^{2} D}$ if and only if $\varphi\left(\epsilon^{k}\right)$ is a scalar matrix $\bmod N$, and since $\operatorname{det}(\varphi(\epsilon))=\operatorname{Norm}(\epsilon)=1$, this is if and only if $\varphi\left(\epsilon^{k}\right)^{-1}=\varphi\left(\epsilon^{-k}\right)$ is a scalar matrix $\bmod N$, which is equivalent to $\epsilon^{-k} \in$ $\mathcal{O}_{N^{2} D}$. We infer that $\epsilon^{k} \in \mathcal{O}_{N^{2} D}$ implies that $\epsilon^{k} \in \mathcal{O}_{N^{2} D}^{\times}$. After noting that $\operatorname{det}(\varphi(\epsilon))=\operatorname{Norm}(\epsilon)= \pm 1$ implies that whenever $\varphi\left(\epsilon^{l}\right)$ is a scalar matrix $\bmod N$ for some $l$, it is in fact $\pm I \bmod N$, (3.1) follows, and therefore also the first part of the corollary.

It is clear that $R(N)$, up to possibly a factor of 2 , is $\operatorname{ord}_{N}(\varphi(\epsilon))$ so the second part also follows.

3.2. Studying $\operatorname{ord}_{N}\left(\varphi\left(\epsilon_{D}\right)\right)$. Let us review and fix some notations: As before, for a matrix $M \in \mathrm{SL}_{2}(\mathbb{Z})$, $\operatorname{ord}_{N}(M)$ is the order of the reduction mod $N$ of $M$ in $\mathrm{SL}_{2}(\mathbb{Z} / N \mathbb{Z})$. Similarly, for $a \in \mathcal{O}_{D}^{\times}$we denote by $\operatorname{ord}_{N}(a)$ the order of $a$ in $\left(\mathcal{O}_{D} / N \mathcal{O}_{D}\right)^{\times}$. The following observation is very simple but crucial to us so we enunciate it in a lemma:

Lemma 9. For all $N \in \mathbb{N}$, $\operatorname{ord}_{N}\left(\varphi\left(\epsilon_{D}\right)\right)=\operatorname{ord}_{N}\left(\epsilon_{D}\right)$.

Proof. Note that $a \in \mathcal{O}_{D}^{\times}$represent the trivial element of $\left(\mathcal{O}_{D} / N \mathcal{O}_{D}\right)^{\times}$if and only if $a=1 \bmod N \mathcal{O}_{D}$. We have $\epsilon_{D}^{k}=1 \bmod N \mathcal{O}_{D}$ if and only if $\epsilon_{D}^{k}=1+N \alpha, \alpha \in \mathcal{O}_{D}$ if and only if $\varphi\left(\epsilon_{D}\right)^{k}=I+N \varphi(\alpha), \alpha \in \mathcal{O}_{D}$ if and only if $\varphi\left(\epsilon_{D}\right)^{k}=I \bmod N$, where the last "if" follows from the fact that $\varphi$ is a field embedding of $K$ and since $\varphi^{-1}\left(M_{2}(\mathbb{Z})\right)=\mathcal{O}_{D}$. 
The study of $\operatorname{ord}_{\mathrm{N}}\left(\epsilon_{\mathrm{D}}\right)$, at least for prime moduli, can be considered as a quadratic analogue of Artin's conjecture on primitive roots [3]. This conjecture asserts that any integer $a \neq \pm 1$ is a primitive root in $(\mathbb{Z} / p \mathbb{Z})^{\times}$for a set of primes of some explicitly given positive density. Under the Generalized Riemann Hypothesis (abbreviated henceforth as GRH), this conjecture has been verified by Hooley [13]. Unconditionally, with some restrictions (analogous to the exceptions in Theorem 10) Gupta and Murty [9] and Heath-Brown's [12] find an infinite set of primes for which $a$ is primitive root modulo these primes. All the results below are various analogues of these works and their proofs are very similar in spirit.

Although we are not using it in the sequel, it is important for us to mention the following quadratic analogue of Hooley's result [13] on Artin's conjecture that was obtained by Roskam [18]:

Theorem. Let $P$ denote the set of prime numbers. Under the GRH, there exists a subset $S=S(D) \subset P$ of positive (explicitly given) density with $\operatorname{ord}_{p}\left(\epsilon_{D}\right)$ is maximal amongst all elements of $\left(\mathcal{O}_{D} / p \mathcal{O}_{D}\right)^{\times}$whenever $p \in S$. In particular,

$$
p \in S \Longrightarrow \operatorname{ord}_{p}\left(\epsilon_{D}\right) \gg p .
$$

Narkiewic [16] proved a quadratic analogue of the results of Gupta and Murty [9] and Heath-Brown's [12] on Artin's Conjecture:

Theorem 10. Unconditionally, with the possible exception of two discriminants $D$, there exists an infinite subset $S=S(D)$ of the primes numbers with $\operatorname{ord}_{p}\left(\epsilon_{D}\right)$ is maximal amongst all elements of $\left(\mathcal{O}_{D} / p \mathcal{O}_{D}\right)^{\times}$whenever $p \in S$. In particular,

$$
p \in S \Longrightarrow \operatorname{ord}_{p}\left(\epsilon_{D}\right) \gg p .
$$

Remark 11. In order to have a better understanding of the bound $\gg p$, it is informative to understand the maximal possible order that $\epsilon_{D}$ can have in $\left(\mathcal{O}_{D} / p \mathcal{O}_{D}\right)^{\times}$. Let $p$ be a rational odd prime which is unramified in $\mathcal{O}_{D}$. If $p$ splits in $D$ then $\mathcal{O}_{D} / p \mathcal{O}_{D} \cong \mathbb{F}_{p} \times \mathbb{F}_{p}$ so $\left(\mathcal{O}_{D} / p \mathcal{O}_{D}\right)^{\times} \cong \mathbb{F}_{p}^{\times} \times \mathbb{F}_{p}^{\times}$. So the maximal order of elements in $\left(\mathcal{O}_{D} / p \mathcal{O}_{D}\right)^{\times}$is $p-1$. When $p$ is inert in $\mathcal{O}_{D}$, we have that $\mathcal{O}_{D} / p \mathcal{O}_{D} \cong \mathbb{F}_{p^{2}}$. If $\operatorname{Norm}_{\mathrm{K} / \mathbb{Q}}\left(\epsilon_{D}\right)=1$ then the image of $\epsilon_{D}$ belongs to the kernel of the norm map from $\mathbb{F}_{p^{2}}^{\times} \rightarrow \mathbb{F}_{p}^{\times}$which is a cyclic group of order $\frac{p^{2}-1}{p-1}=p+1$. Similarly if $\operatorname{Norm}_{K / \mathbb{Q}}\left(\epsilon_{D}\right)=-1$, then the image of $\epsilon_{D}$ belongs to a cyclic group of order $2(p+1)$. In all cases the maximal possible order is $\gg p$.

In fact, the set $S(D)$ of Theorem 10 consists of rational primes that split in $\mathbb{Q}(\sqrt{D})$ so Theorem[10] is true as stated. Joseph Cohen [4] proved a similar result for non-split primes under some more (rather mild) restrictions on the possible discriminants that should be excluded.

Relaxing mildly the condition of being maximal, Par Kurlberg [14 was able to prove a much stronger conditional theorem: 
Theorem 12. Assume the GRH holds and let $M$ be a hyperbolic matrix in $\mathrm{SL}_{2}(\mathbb{Z})$. Then, for every $\epsilon>0$, there exists a subset $S \subset \mathbb{N}$ of density one such that

$$
N \in S \Longrightarrow \operatorname{ord}_{N}(M) \gg_{\epsilon} N^{1-\epsilon} .
$$

Similarly, for every $\epsilon>0$, there exists a subset $T \subset$ Primes of density one such that

$$
p \in T \Longrightarrow \operatorname{ord}_{p}(M) \gg_{\epsilon} p^{1-\epsilon} .
$$

3.2.1. Hecke neighbors. The results of the previous section deal with properties of $x_{D}$ (and correspondingly with $\epsilon_{D}$ ), but we are interested in a general element $x \in K$ which is not necessarily $x_{D}$. To this end we first show that any two elements $K$ can be connected to each other using the Hecke correspondence and then that this influences the regulator and the discriminant only by a multiplicative factor.

Let $p$ be a rational prime; two elements $x, y \in K \backslash \mathbb{Q}$ are called $p$-Hecke neighbors if $\Lambda_{x} \subset \Lambda_{y}$ or $\Lambda_{y} \subset \Lambda_{x}$ with index $p$.

Lemma 13. Let $x, y \in K \backslash \mathbb{Q}$. Then $y$ can be connected to $x$ via a chain of Hecke neighbors. This is, there exist $k \in \mathbb{N}$ and

$$
x_{0}=x, x_{1}, \cdots, x_{k}=y
$$

such that $x_{i}$ and $x_{i+1}$ are $p_{i}$-Hecke neighbors, with $p_{i}=p_{i}(x, y)$ some rational primes.

Proof. Write $y=\frac{A x+B}{C}$ with $A, B, C \in \mathbb{Z}$. Factoring $A$ into primes, we see that $x$ can be connected to $A x$. Clearly $\Lambda_{A x}=\Lambda_{A x+B}$, and by factoring $C$ into primes we see that $A x+B$ can be connected to $\frac{A x+B}{C}=y$.

Lemma 14. Let $x, y \in K \backslash \mathbb{Q}$ be $p$-Hecke neighbors. Then $\operatorname{Reg}\left(\mathcal{O}_{x}\right) \asymp_{p}$ $\operatorname{Reg}\left(\mathcal{O}_{y}\right)$ and $\operatorname{Disc}\left(\mathcal{O}_{x}\right) \asymp_{p} \operatorname{Disc}\left(\mathcal{O}_{y}\right)$.

Proof. Without less of generality we can assume that $\Lambda_{y} \subset \Lambda_{x}$. Note that any element $\alpha \in K^{\times}$act by multiplication as an automorphism on $(K,+)$. Thus any element $\alpha \in \mathcal{O}_{x}^{\times}$fixes $\Lambda_{x}$ and acts on its sublattices of index $p$. Therefore there exists $1 \leq \ell \leq p+1(p+1$ being the number of index $p$ sublattices) such that $\alpha^{\ell}$ fixes $\Lambda_{y}$, i.e., $\alpha^{\ell} \in \mathcal{O}_{y}^{\times}$. Vice versa, any element $\alpha \in \mathcal{O}_{y}^{\times}$fixes $\Lambda_{y}$ and acts on its suplattices (that is, lattices with $\Lambda \supset \Lambda_{y}$ ) of index $p$. As there are also $p+1$ such suplattices, there exists $1 \leq \tilde{\ell} \leq p+1$ such that $\alpha^{\tilde{\ell}}$ fixes $\Lambda_{x}$, i.e., $\alpha^{\tilde{\ell}} \in \mathcal{O}_{x}^{\times}$.

Now, for any $z \in K \backslash \mathbb{Q}$,

$$
\operatorname{Reg}\left(\mathcal{O}_{z}\right)=\operatorname{Reg}\left(\mathcal{O}_{D}\right) \cdot\left(\mathcal{O}_{D}^{\times}: \mathcal{O}_{z}^{\times}\right),
$$

so we have that $\operatorname{Reg}\left(\mathcal{O}_{z}\right) \asymp k(z)$ where $k(z)$ is defined to be the integer such that $\mathcal{O}_{z}^{\times}=\left\langle \pm \epsilon_{D}^{k(z)}\right\rangle$. By the preceding discussion, $k(x) \asymp_{p} k(y)$ so the first part of the lemma follows.

For the second part, first recall (see e.g. [5, Lemma 7.2]) that orders in quadratic fields are monogenic (i.e. of the form $\mathbb{Z}[\alpha]$ ) and since every order 
is contained in $\mathbb{Z}\left[x_{D}\right]$ we can define $l_{z}$ to be the integer with the property $\mathcal{O}_{z}=\mathbb{Z}\left[l_{z} x_{D}\right]$.

Using this, the claim will follows once we show that $l_{x} \asymp_{p} l_{y}$. To this end recall the definition of $\mathcal{O}_{x}$ and note that

$$
l_{z}=\operatorname{gcd}\left\{m: m x_{D} \in \mathcal{O}_{z}\right\} .
$$

Still working under the assumption that $\Lambda_{y} \subset \Lambda_{x}$ and using the fact that any index $p$ sublattice of a lattice $\Lambda$ contains $p \Lambda$, we have:

$$
p l_{x} x_{D} \Lambda_{y} \subset p l_{x} x_{D} \Lambda_{x} \subset p \Lambda_{x} \subset \Lambda_{y}
$$

so (3.2) implies that $l_{y} \mid p l_{x}$. Similarly,

$$
p l_{y} x_{D} \Lambda_{x}=l_{y} x_{D} p \Lambda_{x} \subset l_{y} x_{D} \Lambda_{y} \subset \Lambda_{y} \subset \Lambda_{x}
$$

so $l_{x} \mid p l_{y}$. Therefore $\frac{1}{p} l_{y} \leq l_{x} \leq p l_{y}$. As for any $l \in \mathbb{N}, \operatorname{disc}\left(\mathbb{Z}\left[l x_{D}\right]\right)=l^{2} D$, we are done.

\subsection{Proofs of Theorems 2 and 1 .}

Proof. The proofs of both theorems are very similar. We start with Theorem 2. and comment afterwards on how to adapt the proof for Theorem 1, Let $x$ be the quadratic irrational that appears in the statement of the Theorem and let $D$ be the discriminant of $\mathbb{Q}(x)$. We first translate the statement on continued fractions to a statement on equidistribution of closed geodesics on $X_{2}$ : By [2, Theorem 8.8, Theorem 8.9 and Corollary 2.10] it is enough to show that there exists a density one subset $S=\left\{N_{i}\right\} \subset \mathbb{N}$ and $0<\delta_{1}$ such that for $f \in C_{c}^{\infty}(X)$ we have

$$
\left|\mu_{L_{N_{i} x}}(f)-\mu_{X_{2}}(f)\right| \ll S(f) \operatorname{Disc}\left(L_{N_{i} x}\right)^{-\delta_{1}} .
$$

To this end, we will show that there exists a density one subset $S=\left\{N_{i}\right\} \subset \mathbb{N}$ such that for every $\epsilon>0$ small enough

$$
\operatorname{Reg}\left(\mathcal{O}_{N_{i} x}\right)=l\left(L_{N_{i} x}\right) \gg \operatorname{Disc}\left(L_{N_{i} x}\right)^{\frac{1}{2}-\epsilon} .
$$

This is indeed enough as by Theorem 5, we have that (3.4) implies (3.3).

Toward establishing (3.4), note that there exist

$$
x_{0}=x, x_{1}, \cdots, x_{k}=x_{D}
$$

such that $x_{i}$ and $x_{i+1}$ are $p_{i}$-Hecke neighbors, with $p_{i}=p_{i}(x)$ some rational primes. Recursive use of Lemma 14 implies that $\operatorname{Reg}\left(\mathcal{O}_{x}\right) \asymp_{c(x)} \operatorname{Reg}\left(\mathcal{O}_{x_{D}}\right)$ and $\operatorname{Disc}\left(\mathcal{O}_{x}\right) \asymp_{c(x)} \operatorname{Disc}\left(\mathcal{O}_{x_{D}}\right)$ where $c(x)$ is a constant depending only on $x$. In fact, $c(x)$ is proportional to the product of the rationals primes appearing in the chain of Hecke neighbors connecting $x$ and $x_{D}$. Noting that for every $N \geq 1$, the same rational primes connect $N x$ to $N x_{D}$, it follows that that

\footnotetext{
${ }^{1}$ Note that for different reasons the results in 2 are phrased for Lipschitz functions. For the purpose of this paper, one can easily approximate characteristic functions on $[0,1]$ with smooth functions with compact support, and translate the corresponding statement using the results in 2 .
} 
$\operatorname{Reg}\left(\mathcal{O}_{N x}\right) \asymp_{c(x)} \operatorname{Reg}\left(\mathcal{O}_{N x_{D}}\right)$ and $\operatorname{Disc}\left(\mathcal{O}_{N x}\right) \asymp_{c(x)} \operatorname{Disc}\left(\mathcal{O}_{N x_{D}}\right)$, that is, the proportionality constant $c(x)$ is independent of $N$. It follows that it is enough to show (3.4) for $x_{D}$ instead of $x$ so we can use the results of Subsection 3.2 which implicitly deals with $x=x_{D}$.

Assuming GRH and applying Theorem 12 for the hyperbolic matrix $\varphi\left(\epsilon_{D}\right)$ and Corollary 8 , we find a density one subset $S=\left\{N_{i}\right\} \subset \mathbb{N}$ such that for every $\epsilon>0$ small enough we have

$$
\operatorname{Reg}\left(\mathcal{O}_{N_{i}^{2} D}\right) \gg \operatorname{ord}_{\mathrm{N}_{\mathrm{i}}}\left(\varphi\left(\epsilon_{\mathrm{D}}\right)\right) \gg_{\epsilon} N_{i}^{1-\epsilon} .
$$

Note that $\Lambda_{N x_{D}}=\mathbb{Z} \cdot 1 \oplus \mathbb{Z} \cdot N x_{D}$ is in fact the order $\mathbb{Z}\left[N x_{D}\right]$, so $\mathcal{O}_{N_{i} x_{D}}=$ $\mathbb{Z}\left[N_{i} x_{D}\right]$ which has discriminant $N_{i}^{2} D$. Thus $\operatorname{Reg}\left(\mathcal{O}_{L_{N_{i} x_{D}}}\right)=\operatorname{Reg}\left(\mathcal{O}_{N_{i}^{2} D}\right)$ and $\operatorname{Disc}\left(\mathcal{O}_{L_{N_{i} D} D}\right)=\operatorname{Disc}\left(\mathcal{O}_{N_{i}^{2} D}\right)=N_{i}^{2} D$. Therefore (3.5) implies (3.4) for $x=x_{D}$ (where the implicit constants depend only on $x$, which is fixed throughout). This concludes the proof of the Theorem 2

The proof of the second part of Theorem 2 follow the exact same lines using as an input the second part of Theorem 12 .

We explain how to adapt the proof above to get a proof for Theorem 1. Assuming $D$ does not fall into the 2 possible exceptions, Theorem 10 furnishes an infinite set of primes $S=\left\{p_{i}\right\}$ with

$$
\operatorname{ord}_{p_{i}}\left(\epsilon_{D}\right) \gg p_{i} \text {. }
$$

Translating through Lemma 9 and using Corollary 8 we see that

$$
\operatorname{Reg}\left(\mathcal{O}_{p_{i}^{2} D}\right) \gg \operatorname{ord}_{p_{i}}\left(\varphi\left(\epsilon_{D}\right)\right) \gg_{\epsilon} p_{i}
$$

which establish a (slightly stronger) analog of (3.5). One proceeds now exactly as above to establish Theorem 1 .

\section{REFERENCES}

[1] Menny Aka and Manfred Einsiedler. Duke's theorem for subcollections. Ergodic Theory Dynam. Systems, 36(2):335-342, 2016.

[2] Menny Aka and Uri Shapira. On the evolution of continued fractions in a fixed quadratic field. J. Anal. Math., 134(1):335-397, 2018.

[3] Emil Artin. The collected papers of Emil Artin. Edited by Serge Lang and John T. Tate. Addison-Wesley Publishing Co., Inc., Reading, Mass.-London, 1965.

[4] Joseph Cohen. Primitive roots in quadratic fields. Int. J. Number Theory, 2(1):7-23, 2006.

[5] David A Cox. Primes of the form x2+ ny2: Fermat, class field theory, and complex multiplication, volume 34. John Wiley \& Sons, 2011.

[6] Manfred Einsiedler, Elon Lindenstrauss, Philippe Michel, and Akshay Venkatesh. Distribution of periodic torus orbits on homogeneous spaces. Duke Math. J., 148(1):119$174,2009$.

[7] Manfred Einsiedler, Elon Lindenstrauss, Philippe Michel, and Akshay Venkatesh. The distribution of closed geodesics on the modular surface, and Duke's theorem. Enseign. Math. (2), 58(3-4):249-313, 2012.

[8] Manfred Einsiedler and Thomas Ward. Ergodic theory with a view towards number theory, volume 259 of Graduate Texts in Mathematics. Springer-Verlag London, Ltd., London, 2011. 
[9] Rajiv Gupta and M. Ram Murty. A remark on Artin's conjecture. Invent. Math., 78(1):127-130, 1984.

[10] Gergely Harcos. Subconvex bounds for automorphic l-functions and applications. This is an unpublished dissertation available at http://www.renyi.hu/ gharcos/ertekezes.pdf.

[11] Gergely Harcos and Philippe Michel. The subconvexity problem for Rankin-Selberg $L$-functions and equidistribution of Heegner points. II. Invent. Math., 163(3):581-655, 2006.

[12] D. R. Heath-Brown. Artin's conjecture for primitive roots. Quart. J. Math. Oxford Ser. (2), 37(145):27-38, 1986.

[13] Christopher Hooley. On Artin's conjecture. J. Reine Angew. Math., 225:209-220, 1967.

[14] Pär Kurlberg. On the order of unimodular matrices modulo integers. Acta Arith., 110(2):141-151, 2003.

[15] Philippe Michel and Akshay Venkatesh. Equidistribution, L-functions and ergodic theory: on some problems of Yu. Linnik. In International Congress of Mathematicians. Vol. II, pages 421-457. Eur. Math. Soc., Zürich, 2006.

[16] W. Narkiewicz. Units in residue classes. Arch. Math. (Basel), 51(3):238-241, 1988.

[17] Alexandru A. Popa. Central values of Rankin $L$-series over real quadratic fields. Compos. Math., 142(4):811-866, 2006.

[18] Hans Roskam. A quadratic analogue of Artin's conjecture on primitive roots. J. Number Theory, 81(1):93-109, 2000.

ETH ZÜrICH, RÄmistrasse 101, CH-8092 ZÜrich, Switzerland

E-mail address: mennyaka@math.ethz.ch 